\title{
Oligodendrogliopathy in Multiple Sclerosis: Low Glycolytic Metabolic Rate Promotes Oligodendrocyte Survival
}

\author{
Malena B. Rone, ${ }^{1 \star}$ Qiao-Ling Cui, ${ }^{1 \star}$ Jun Fang, ${ }^{2}$ - Li-Chun Wang, ${ }^{2}$ Ji Zhang, ${ }^{1,3}$ Damla Khan, ${ }^{1}$ Melissa Bedard, ${ }^{1}$ \\ Guillermina Almazan, ${ }^{2}$ Samuel K. Ludwin, ${ }^{1}$ Russel Jones, ${ }^{4}$-Timothy E. Kennedy, ${ }^{1}$ and Jack P. Antel ${ }^{1}$ \\ ${ }^{1}$ Department of Neurology and Neurosurgery, Montreal Neurological Institute, McGill University, Montreal, Quebec H3A 2B4, Canada, ${ }^{2}$ Department of \\ Pharmacology and Therapeutics, McGill University, Montreal, Quebec H3G 1Y6, Canada, and ${ }^{3}$ Alan Edwards Centre for Research on Pain and ${ }^{4}$ Department \\ of Physiology, McGill University, Montreal, Quebec H3A 0G4, Canada
}

Multiple sclerosis (MS) lesions feature demyelination with limited remyelination. A distinct injury phenotype of MS lesions features dying back of oligodendrocyte $(\mathrm{OL})$ terminal processes, a response that destabilizes myelin/axon interactions. This oligodendrogliopathy has been linked with local metabolic stress, similar to the penumbra of ischemic/hypoxic states. Here, we developed an in vitro oligodendrogliopathy model using human CNS-derived OLs and related this injury response to their distinct bioenergetic properties. We determined the energy utilization properties of adult human surgically derived OLs cultured under either optimal or metabolic stress conditions, deprivation of growth factors, and glucose and/or hypoxia using a Seahorse extracellular flux analyzer. Baseline studies were also performed on OL progenitor cells derived from the same tissue and postnatal rat-derived cells. Under basal conditions, adult human OLs were less metabolically active than their progenitors and both were less active than the rat cells. Human OLs and progenitors both used aerobic glycolysis for the majority of ATP production, a process that contributes to protein and lipid production necessary for myelin biosynthesis. Under stress conditions that induce significant process retraction with only marginal cell death, human OLs exhibited a significant reduction in overall energy utilization, particularly in glycolytic ATP production. The stress-induced reduction of glycolytic ATP production by the human OLs would exacerbate myelin process withdrawal while favoring cell survival, providing a potential basis for the oligodendrogliopathy observed in MS. The glycolytic pathway is a potential therapeutic target to promote myelin maintenance and enhance repair in MS.

Key words: aerobic glycolysis; metabolic stress; multiple sclerosis; oligodendrocytes; oligodendrogliopathy

Significance Statement

The neurologic deficits that characterize multiple sclerosis (MS) reflect disruption of myelin (demyelination) within the CNS and failure of repair (remyelination). We define distinct energy utilization properties of human adult brain-derived oligodendrocytes and oligodendrocyte progenitor cells under conditions of metabolic stress that model the initial relapsing and subsequent progressive phases of MS. The observed changes in energy utilization affect both cell survival and myelination capacity. These processes may be amenable to therapeutic interventions to limit the extent of cumulative tissue injury and to promote repair in MS.

\section{Introduction}

Multiple sclerosis (MS) lesions feature demyelination with a variable but usually limited extent of remyelination, even in the pres-

\footnotetext{
Received Nov. 11, 2015; revised Jan. 28, 2016; accepted Feb. 21, 2016.

Author contributions: M.B.R. and Q.-L.C. designed research; M.B.R., Q.-L.C., J.F., L.-C.W., and M.B. performed research; M.B.R., Q.-L.C., and J.Z. contributed unpublished reagents/analytic tools; M.B.R., Q.-L.C., D.K., G.A., S.K.L., R.J., T.E.K., and J.P.A. analyzed data; M.B.R., Q.-L.C., J.Z., G.A., S.K.L., R.J., T.E.K., and J.P.A. wrote the paper.

This work was supported by an operating grant from the MS Society of Canada. M.B.R. was supported by a postdoctoral fellowship from the MS Society of Canada.

The authors declare no competing financial interests.

*M.B.R. and Q.-L.C. contributed equally to this study.

Correspondence should be addressed to Jack P. Antel, Neuroimmunology Unit, Montreal Neurological Institute,

McGill University, 3801 University Street, Montreal, Quebec H3A 2B4, Canada. E-mail: jack.antel@mcgill.ca.
}

ence of remaining oligodendrocytes (OLs) and OL progenitor cells (OPCs) (Chang et al., 2000). Pathologic analyses of acute MS lesions indicate heterogeneity of the injury response (Lucchinetti et al., 2000). Such injury has been attributed to both a systemic immune system-derived attack on OLs/myelin, as modeled by studies in experimental autoimmune encephalolmyelitis, and to oligodendrogliopathy (Trapp and Nave, 2008). The latter features preservation of the OL cell body with dying back of terminal processes that destabilizes the compact axonal myelin sheath, a 


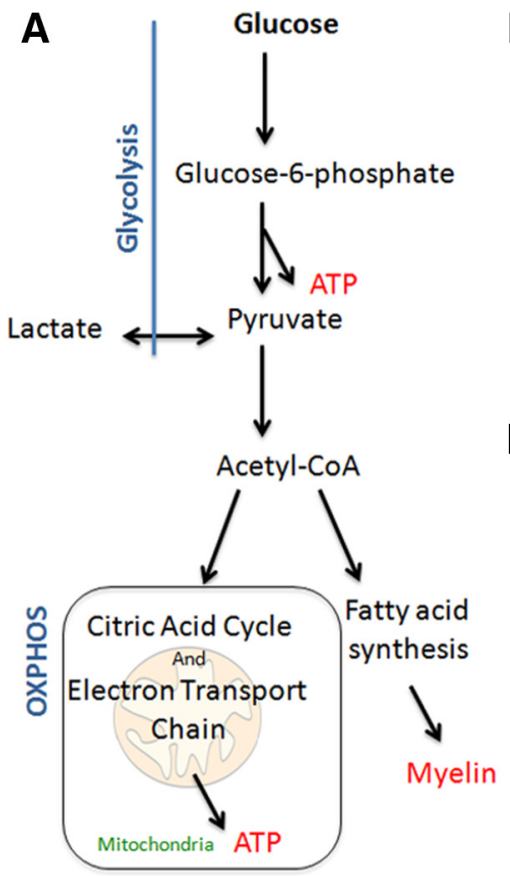

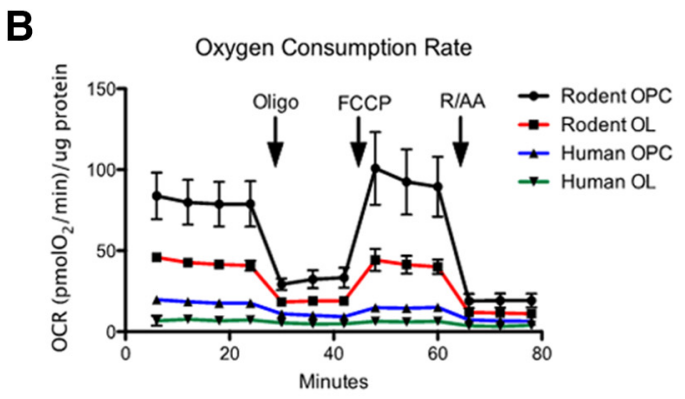

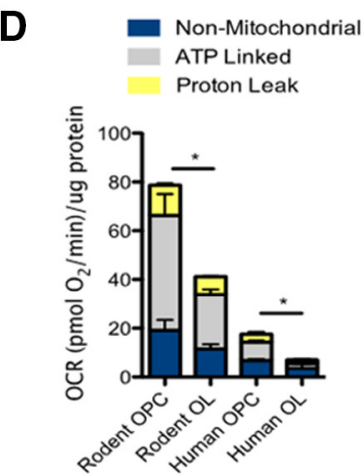

E ATP Production OXPHOS

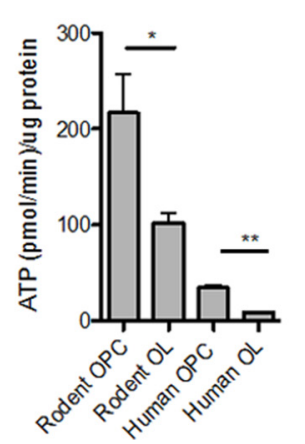

C

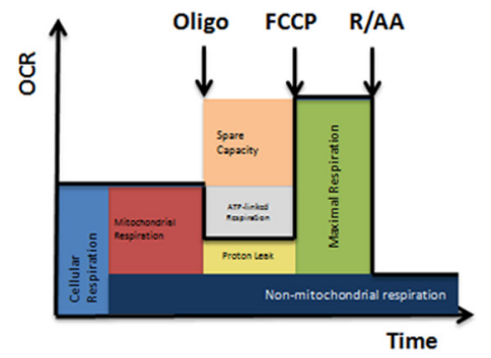

$F$

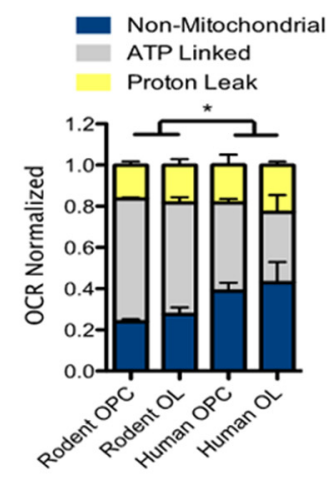

Figure 1. Distinct oxidative phosphorylation properties of human OLs and OPCS. A, Pathway depicting glucose metabolism in $0 \mathrm{Ls}$. Glucose metabolism via the glycolytic pathway results in two molecules of ATP produced and pyruvate. Pyruvate can be converted into lactate and exported out of the cell. Continued metabolism of pyruvate into acetyl-CoA can be used either in ATP production via OXPHOS, generating 34 molecules of ATP, or in fatty acid biosynthesis to be used in myelin production. $\boldsymbol{B}$, Cellular OCRs of rodent and human OLs and OPCs were measured using an XF96 extracellular flux analyzer. C, Scheme demonstrating breakdown of oxygen consumption after the addition of the stated inhibitors. The addition of Oligo identifies ATP-linked oxygen consumption (gray bar), whereas the addition of mitochondrial ETC inhibitors $\mathrm{R}$ and AA allowed for the identification of nonmitochondrial (blue bar) $0 C R$ with the difference between the two identifying mitochondrial proton leak (yellow bar). The addition of FCCP identifies mitochondrial spare capacity. $D$, Human and rodent OPCs had significantly higher basal oxygen consumption, nonmitochondrial oxygen, and ATP-linked OCR than their OL counterparts. $E$, There was a significant increase in rodent proton leak in OPCs compared with $0 \mathrm{Ls}(p<0.05)$, with no differences observed in human proton leak ATP produced via OXPHOS was significantly greater in rodent cells than in their human counterparts $\left(0 \mathrm{PCs},{ }^{*} p<0.01 ; 0 \mathrm{Ls},{ }^{* *} p<0.001\right)$ and in $0 \mathrm{PCS}$ compared with $0 \mathrm{Ls}$. $\boldsymbol{F}$, Normalization of $O C R$ demonstrates that human $0 \mathrm{Ls}$ and $\mathrm{OPC}$ consume a significantly higher quantity of oxygen for nonmitochondrial activities $\left({ }^{*} p<0.05\right)$, with resulting lower ATP-linked $0 C R$ compared with rodent $\mathrm{OL} / \mathrm{OPCS}$.

process detrimental to neuronal function (Ludwin and Johnson, 1981; Rodriguez et al., 1993). In situ analysis of MS oligodendrogliopathy has been associated with evidence of local ischemia/ hypoxia (Stadelmann et al., 2005; Trapp and Stys, 2009) and these features have also been observed in the penumbra of white matter ischemic lesions (Aboul-Enein et al., 2003). This altered metabolic profile of local ischemia/hypoxia is also present in the progressive phase of MS and is speculated to involve continued metabolic and immune injury of damaged OLs (Witte et al., 2014; Mahad et al., 2015). A metabolic state resembling hypoxia could be induced in inflammatory MS lesions either by defective microcirculation and subsequent ischemia or by the local production of toxic metabolites that interfere with energy metabolism (Lassmann, 2003).

Currently, little is known about the metabolic profile of OLs and the energetic requirements needed for myelination (Amaral et al., 2013). The biosynthesis of myelin is a highly energetic process (Harris and Attwell, 2012), requiring mitochondrial oxidative phosphorylation (OXPHOS) for ATP production and glycolysis/lactate to provide the carbon backbones necessary for myelin biosynthesis (Sánchez-Abarca et al., 2001; Lunt and Vander Heiden, 2011) (see Fig. 1A). Inhibition of mitochondrial OXPHOS in vitro can alter the initiation of myelin production and increase OPC and OL cell death (Ziabreva et al., 2010). In contrast, inhibiting the OXPHOS pathway in adult rodent OLs in vivo resulted in no cell death or process retraction (Fünfschilling et al., 2012), suggesting different energetic and cellular requirements of OLs and OPCs.
The aim of our study was to define the energy utilization properties of human OLs derived from the adult human CNS under basal conditions and under metabolic stress conditions that produced sublethal injury modeling the oligodendrogliopathy seen in MS. The adult human OLs are postmitotic cells that express all of the markers that would be expected of fully differentiated OL lineage cells (Ruffini et al., 2006). We compared these OLs with OPCs isolated from the same tissue using A2B5 antibody as the selection marker. A majority of OPCs express O4, an OL lineage marker, in contrast to A2B5- or platelet-derived growth factor receptor-alpha (PDGFR $\alpha$ )-expressing cells in the developing CNS (Leong et al., 2014). The adult CNS-derived cells retain the potential for cell division and express distinct miRNA profiles compared with the OLs and with fetal CNS-derived progenitor cells (Leong et al., 2014). Our results showed that, under basal conditions, adult human OLs were less metabolically active than OPCs, consistent with different energetic requirements at different stages of differentiation. Both were less active than OPCs and OLs derived from postnatal rats. The human OLs and OPCs both used glycolysis for the majority of ATP production with little contribution from OXPHOS. Under metabolic stress conditions in which the OLs show process retraction but marginal cell death, they significantly reduced overall energy production rate. In these same conditions, OPCs increase process retraction and cell death, but do not reduce the metabolic rate. These findings provide evidence that energy reduction and process retraction by a myelinating OL may function to promote survival and limit cell loss in response to metabolic stress. This is 
in contrast to OPCs, which are unable to decrease their metabolic rate, resulting in an increase in cell death.

\section{Materials and Methods}

Isolation of adult human OPCs and OLs. Brain tissue was obtained from adults undergoing surgical resections as a treatment for nontumor-related intractable epilepsy in accordance with the guidelines set by the Biomedical Ethics Unit of McGill University. There was no gender bias. The material came predominantly from temporal lobe white matter and did not include subependymal regions. Tissue specimens were enzymatically digested and placed on a linear 30\% Percoll density gradient (Pharmacia Biotech). Microglia were separated and removed by an initial adhesion step in which the total cell fraction was cultured for $24 \mathrm{~h}$ in noncoated flasks. The floating cell fraction was subjected to immunomagnetic bead selection with the A2B5 antibody (IgM), which recognizes gangliosides (Eisenbarth et al., 1979). As described previously (Cui et al., 2013), OPCs $\left(\mathrm{A} 2 \mathrm{~B} 5^{+}\right)$or OLs $\left(\mathrm{A} 2 \mathrm{~B} 5^{-}\right)$were initially plated at a density of $2.5 \times 10^{5}$ cells per milliliter on poly-lysine-coated chamber slides and cultured in defined medium (DFM) consisting of Dulbecco's modified essential medium DMEM-F12 supplemented with N1 (SigmaAldrich), $0.01 \%$ bovine serum albumin, $1 \%$ penicillin-streptomycin, B27 supplement (Invitrogen), PDGF-AA (10 ng/ml), basic fibroblast factor $(10 \mathrm{ng} / \mathrm{ml})$, and triiodothyronine (2 nм) (Sigma-Aldrich) for $4 \mathrm{~d}$. Media used for our subsequent experimental studies included: DFM as the optimal medium, DMEM without supplementation (DMEM) as a less nutritionally enriched medium, and DMEM with $0.25 \mathrm{~g} / \mathrm{L}$ glucose (DMEM/LG) as our most metabolically deprived medium. For all extended studies, media were changed every $48 \mathrm{~h}$.

Rodent OL cultures. All procedures involving animals were performed in accordance with the Canadian Council on Animal Care's guidelines for the use of animals in research. OPCs were prepared from the brains of newborn Sprague Dawley rats as described previously (Almazan et al., 1993) and plated on poly-D-lysine-coated culture dishes grown in the media described for human OPCs and OLs. Removal of mitogens and 1\% newborn calf serum was added to initiate OL differentiation. Culture media were changed every $48 \mathrm{~h}$ with stated conditions until completion of the experiment.

Immunocytochemistry. For assessment of cell surface markers, cells were incubated with primary antibodies $(\mathrm{O} 4)$ for $30 \mathrm{~min}$ at $4^{\circ} \mathrm{C}$, and then fixed in $4 \%$ paraformaldehyde for $10 \mathrm{~min}$ at $4^{\circ} \mathrm{C}$, followed by blocking with HHG (1 mM HEPES, 2\% horse serum, 10\% goat serum, Hanks' balanced salt solution) for $10 \mathrm{~min}$. Cultures were incubated with secondary antibodies conjugated with TexRed for $30 \mathrm{~min}$ at $4^{\circ} \mathrm{C}$. TUNEL ${ }^{+}$cells were identified with a commercial kit (Promega). Cell nuclei were stained with Hoechst 33258 (10 $\mu \mathrm{g} / \mathrm{ml}$; Invitrogen) for $10 \mathrm{~min}$ at room temperature. Cell viability was also assessed by live-staining with propidium iodide (PI; Invitrogen). Further staining was performed with LC3B (Cell Signaling Technology) to measure autophagy. JC-1 Dye (Cayman) was used to assess mitochondrial membrane potential (16606353). Cells were then imaged via MetaXpress High-content image acquisition (Molecular Devices).

Relative process area per cell was calculated from MetaXpress images as total area measured/cell number as determined from ImageJ software version 1.47f. An Olympus VivaView incubator/fluorescence microscope was used to image cell process outgrowth and retraction by longterm time lapse. For these studies, ECM gel (Sigma-Aldrich) was used to

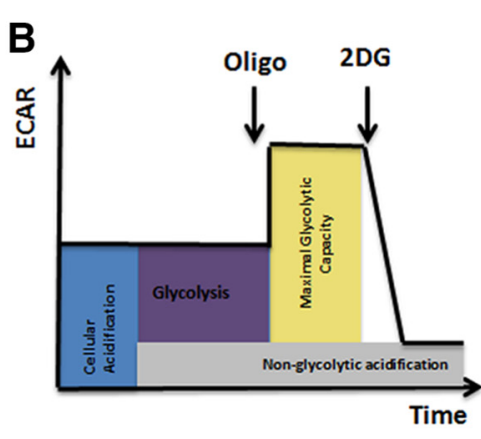

E $\square$ Non-Glycolytic

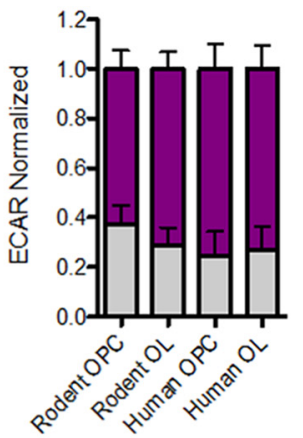

Figure 2. Human and rodent $\mathrm{OPCs}$ are more glycolytic than their $\mathrm{OL}$ counterparts. $A, \mathrm{ECAR}$ as measured concurrently with $O C R$. $B$, scheme demonstrating the breakdown of ECAR after the addition of the stated inhibitors. The addition of 2DG identifies $D$, Calculated glycolytic ATP production revealing no significant difference seen between OLs and OPCS. E, Normalization of ECAR revealing no changes in sources of acidification, demonstrating similar pathway rates.

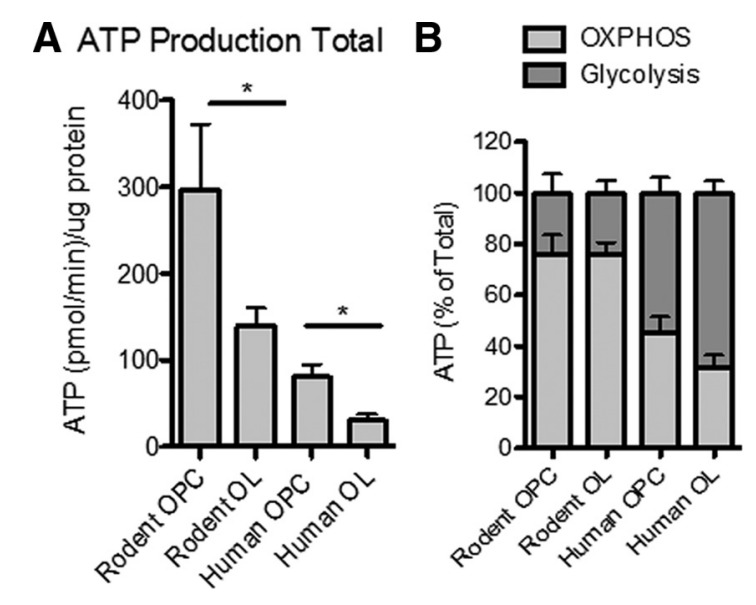

Figure 3. Human $0 \mathrm{Ls}$ and $\mathrm{OPCS}$ produce less ATP than rodent $\mathrm{OLs}$ and $\mathrm{OPCS}$. $A, \mathrm{OL}$ produce significant less ATP than OPCS, with human OPCs and OLs producing less total ATP compared with rodent cells $(p<0.05)$. B, Percentage normalization of ATP production determined that human OLs and OPCs both use the glycolytic pathways significantly more to produce ATP compared with rodent cells $(p<0.05)$.

obtain adequate cell adherence to the $35 \mathrm{~mm}$ glass-bottomed dishes (MatTek).

Metabolic analysis. Rodent OLs and OPCs were treated with DMEM for $24 \mathrm{~h}$; human OLs and OPCs were grown for $48 \mathrm{~h}$ in the stated media conditions. The cells were washed with XF assay medium containing 0.25 $\mathrm{g} / \mathrm{L}$ or $4.5 \mathrm{~g} / \mathrm{L}$ glucose ( $\mathrm{pH}$ adjusted to 7.4 ) and equilibrated for $1 \mathrm{~h}$ in the Seahorse incubator. The XF96 plate was inserted into the Seahorse analyzer, where 4 basal assay cycles were performed consisting of $3 \mathrm{~min}$ of mixing followed by $3 \mathrm{~min}$ of measuring. After completion, oligomycin 

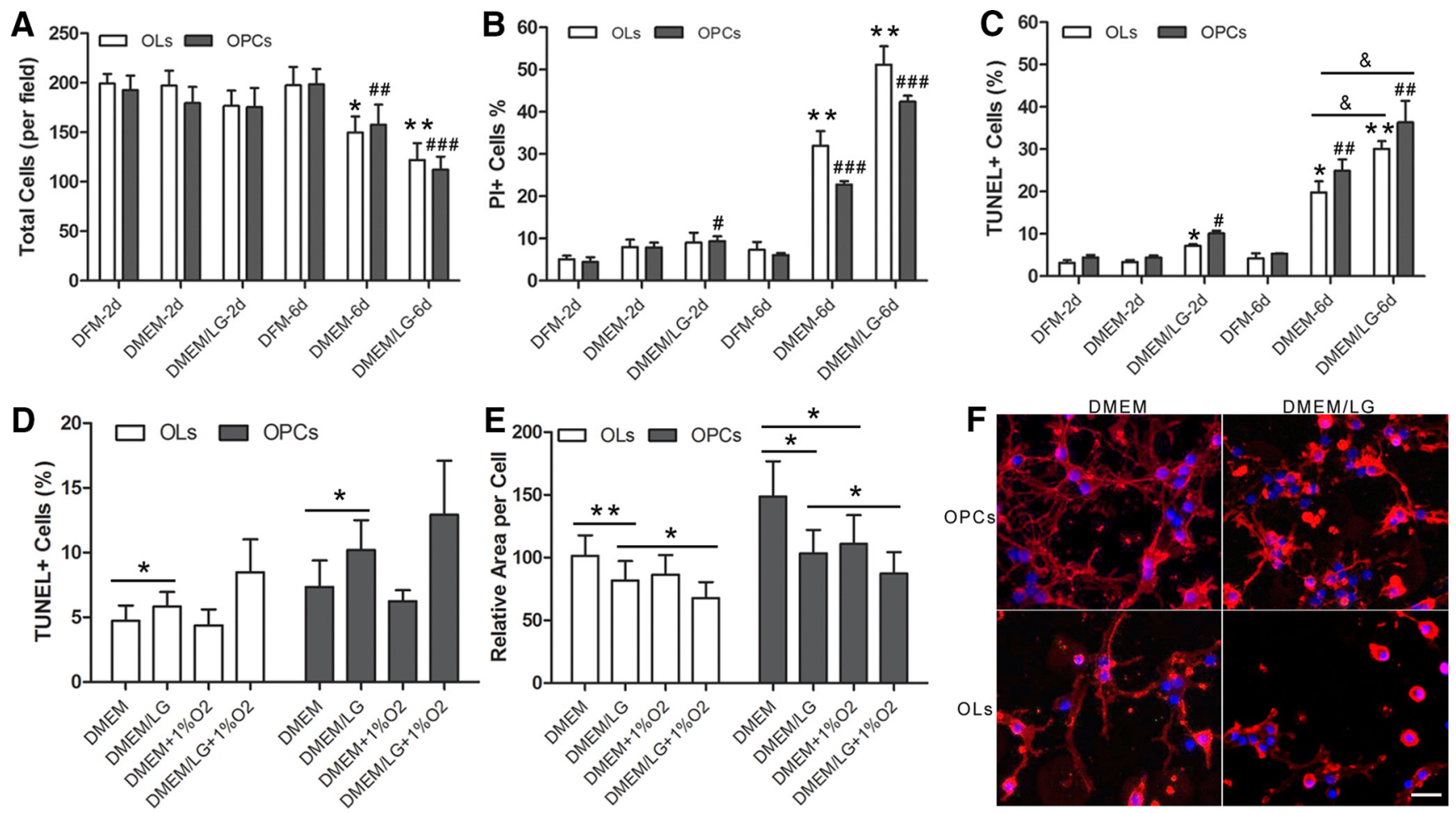

Figure 4. Increased cell death and process retraction in human OLs and OPCs under stress conditions. OLs and OPCs were grown in DFM, DMEM, or DMEM/LG for 2-6 d. $A-C$, Total cell number $(\boldsymbol{A})$, percentage $\mathrm{PI}^{+}(\boldsymbol{B})$, and percentage TUNEL ${ }^{+}(\boldsymbol{C})$ OLs and OPCs were determined. Significant increases in cell death measures under suboptimal conditions are noted at day 6 . For the comparison of OLs in DMEM or DMEM/LG with the OLs in DFM: ${ }^{*} p<0.05$; ${ }^{* *} p<0.01$; for the comparison of OPCs in DMEM or DMEM/LG with the OPCs in DFM: \#p $<0.05$, \#\#p $<0.01$, \#\#\#p $<0.001$; for the comparison between $0 \mathrm{Ls}$ and $\mathrm{OPCs}: \& p<0.05$ (paired $t$ test between groups) $(n=3)$. Human adult $0 \mathrm{Ls}$ and $\mathrm{OPC}$ were grown in DMEM or DMEM/LG \pm hypoxia $\left(1 \% 0_{2}\right)$ for $2 \mathrm{~d} . \boldsymbol{D}$, The percentage of TUNEL ${ }^{+}$cells was marginally increased for both OLs and OPCs under DMEM/LG conditions compared with DMEM. $E$, Relative area per cell was significantly decreased under DMEM/LG and DMEM/LG $/ 1 \% 0_{2}$ conditions compared with DMEM ( ${ }^{*} p<0.05,{ }^{* *} p<0.01$, paired $t$ test between groups, $n=6$ ). $F$, Immunostaining of human 0 Ls and OPCs with 04 antibody and Hoechst 33258 showing the morphological changes (reduced process outgrowth) of cells treated with DMEM/LG versus DMEM for both OLs and OPCs. The costaining with 04 antibody and Hoechst 33258 indicates that the purity of these cell cultures is $>90 \% 04^{+}$cells. Scale bar, $20 \mu \mathrm{m}$.

(0.5 $\mu \mathrm{M}$ injection volume) was added by automatic pneumatic injection for 3 assay cycles. Carbonyl cyanide-4-(trifluoromethoxy) phenylhydrazone (FCCP; $0.5 \mu \mathrm{M}$ ) was then added for an additional three assay cycles, followed by rotenone $(1 \mu \mathrm{M})$ plus AA $(2 \mu \mathrm{M})$ for another three assay cycles. The coupling efficiency to calculate mitochondrial ATP production was calculated from the oxygen consumption rate (OCR) differences upon addition of oligomycin from basal rate, converting the OCR to ATP production using a phosphate/oxygen ratio of 2.3 (Brand, 2005; Divakaruni et al., 2014). Extracellular acidification rate (ECARs) were calculated by the addition of 2-deoxy-glucose (2DG; $1 \mathrm{M})$. The proton production rate was used to estimate ATP production from glycolysis in a 1:1 ratio (Keuper et al., 2014). All reagents were purchased from Sigma-Aldrich

Data analysis. Results are presented as the mean \pm SEM. The number of individual experiments for each study is indicated in the Results section. Statistical significance was determined by Student's $t$ test unless stated otherwise. $p<0.05$ was considered statistically significant.

\section{Results}

\section{Energetic characterization of OLs}

To identify the primary mechanism for energy production in OLs and OPCs, we used a Seahorse XF96 extracellular flux analyzer to assess the metabolic properties of the cell through OCR and ECAR. The OCR can be used to identify the rate of ATP production from oxidative phosphorylation and ECAR determines the ATP produced by glycolysis (Fig. 1A).

\section{OCR}

The basal OCR of human OLs and OPCs was significantly lower than that of their rodent counterparts (Fig. $1 B, D$ ). In addition, in either species, OPCs consumed more oxygen than OLs (Fig. $1 B, D$ ). We then used various mitochondrial inhibitors to understand the mechanism by which the OLs and OPCs consume oxygen (Fig. 1C). OCR due to ATP production was determined by the addition of oligomycin (Oligo), which inhibits mitochondrial ATP-synthase. The Oligo-sensitive OCR of OLs was less than that of their OPC counterparts in both human and rodent samples (Fig. 1D, gray bars), demonstrating that OLs consume less oxygen for ATP production than do OPCs. The calculation of ATP production rate via OXPHOS was determined as stated in the Materials and Methods, confirming the significant decrease in OL ATP production compared with OPCs as was seen in OCR (Fig. 1E). The electron transport chain inhibitors rotenone (R), a complex I inhibitor, and AA, a complex III inhibitor, were added to inhibit remaining mitochondrial oxygen consumption. This inhibition allowed calculation of proton leak (Oligo OCR minus R/AA OCR; Fig. $1 C$ ), identifying a significant difference in rodent OPCs compared with OLs OCR (Fig. $1 B, D$, yellow bar). The OCR remaining after inhibition of mitochondrial OCR allows identification of nonmitochondrial oxygen consumption, which has been speculated to correspond to glycolysis (Herst and Berridge, 2007). We observed no significant changes in nonmitochondrial OCR between OLs and OPCs for either human- or rodentderived cells (Fig. $1 B, D$, blue bar). These results demonstrate that human and rodent OPCs are more metabolically active than OLs, resulting in higher ATP production.

Due to the substantial differences between their basal rates of oxygen consumption, we normalized the OCR of each sample to 

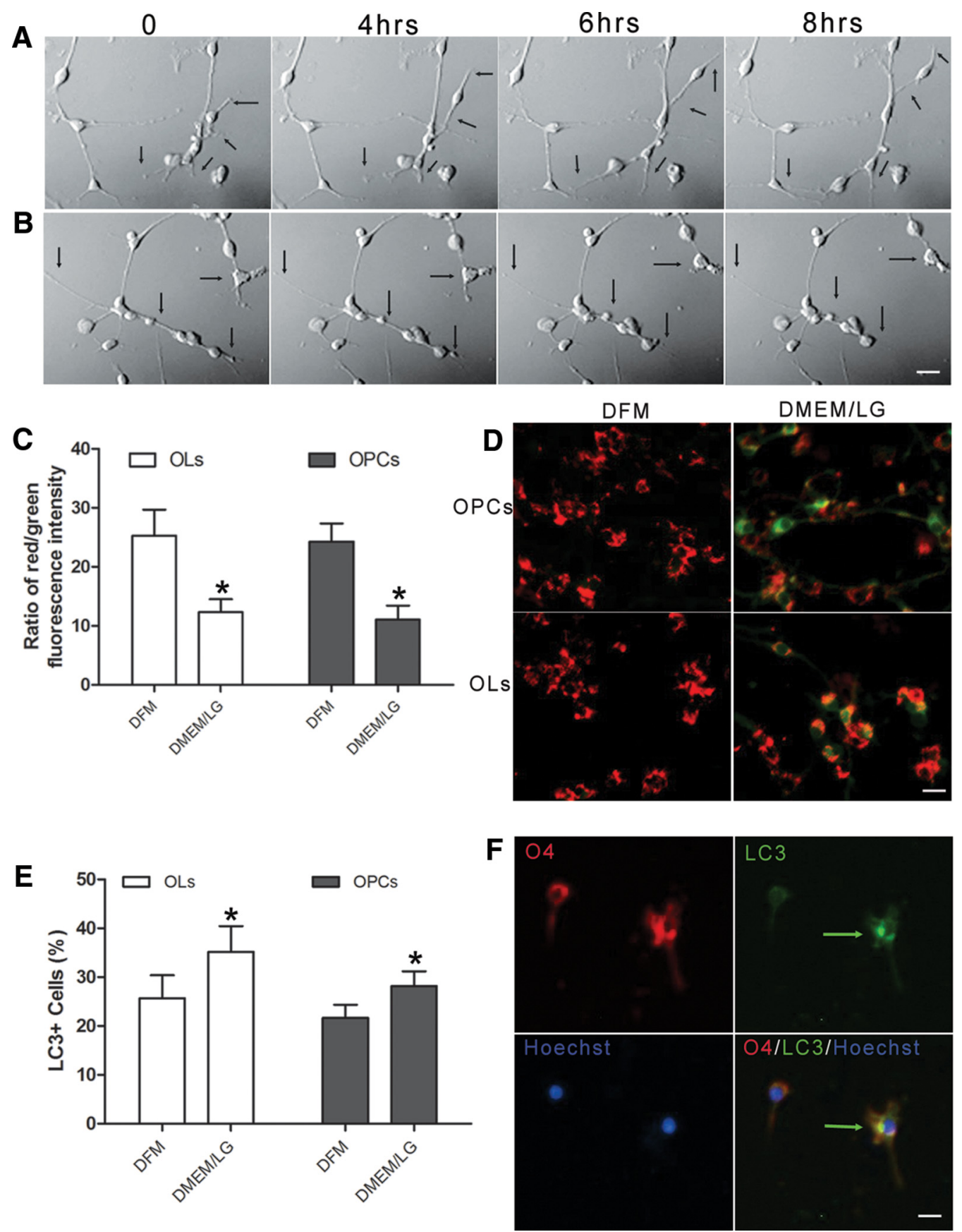

Figure 5. Reduced process outgrowth, mitochondria depolarization, and autophagy activation are induced in human OLs and OPCs under suboptimal conditions. $\boldsymbol{A}, \boldsymbol{B}, \mathrm{L}$ Long-term (8 h) time-lapse imaging of sustained OL process outgrowth under optimal conditions (DFM; $\boldsymbol{A}$ ) and retraction of processes under suboptimal conditions (DMEM/LG; $\boldsymbol{B}$ ) using a VivaView Incubator/fluorescence microscope. Scale bar, $20 \mu \mathrm{M}$. C, D, Reduced ratio of red/green fluorescence intensity of JC-1 dye staining of mitochondria of adult OLs and OPCs grown in DMEM/LG for $48 \mathrm{~h}$ compared with corresponding cells grown in DFM, indicating depolarization of mitochondrial membrane potential. ${ }^{*} p<0.05$ for comparison with corresponding DFM $(n=3)$. Scale bar, $20 \mu \mathrm{m}$. Human OLs and OPCs were grown in DFM or DMEM/LG for $48 \mathrm{~h}$. $E$, Percentage of total cells with LC3B aggregation was increased in DMEM/LG conditions compared with corresponding cells grown in DFM for both OLs and OPCs. F, Illustration of cells immunostained with 04 (red) and LC $3 B$ (green) antibodies. Scale bar, $20 \mu \mathrm{M}$. $p<0.05$ $(n=3)$.

their basal rates, allowing us to determine whether rodents and humans consumed oxygen through a similar mechanism (Fig. $1 F)$. Although proton leak consumed $20 \%$ of total oxygen consumption in all samples, human OLs and OPCs showed a significant increase in nonmitochondrial oxygen consumption compared with rodent cells, which used more oxygen for ATP production. These results verify that human OLs and OPCs use a lower total percentage of oxygen to generate ATP and instead use this oxygen for nonmitochondrial functions.

\section{ECAR}

To assess the glycolytic activity of these cells, we measured the ECAR acquired simultaneously with the OCR. Basal ECAR was lower in OLs than in OPCs for both human and rodent cells (Fig. 2A,C). To determine the amount of cellular acidification that is due to glycolysis, 2DG, a glycolytic inhibitor, was added at the stated time point (Fig. 2A,B). The inhibition of glycolysis allowed calculation of ATP produced from glucose metabolism by measurement of acidification, revealing that, in both rodent and human cells, OPCs exhibit 
A
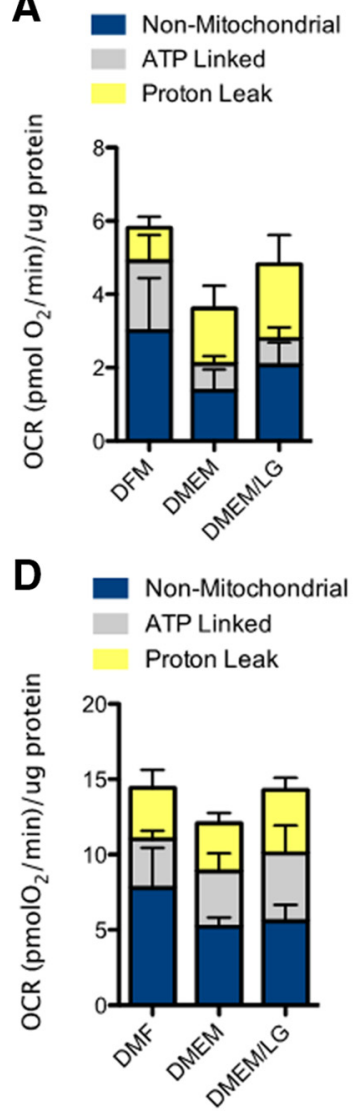

B

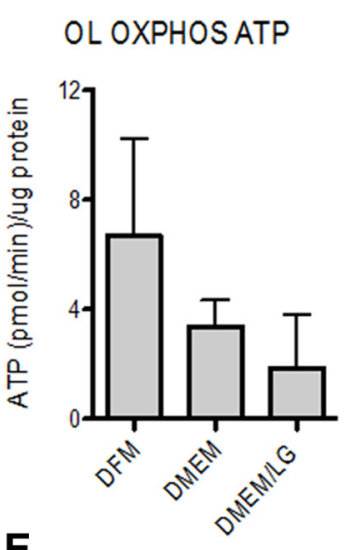

E OPC OXPHOS ATP

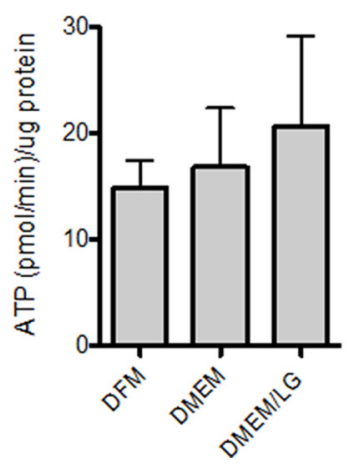

C
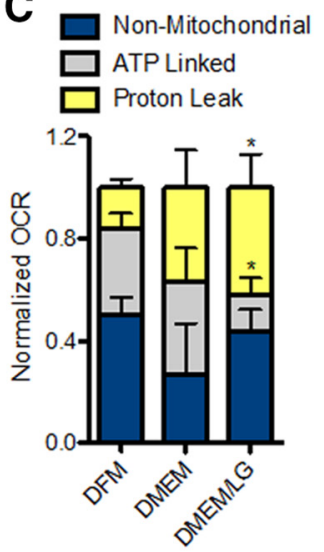

$\mathbf{F}$
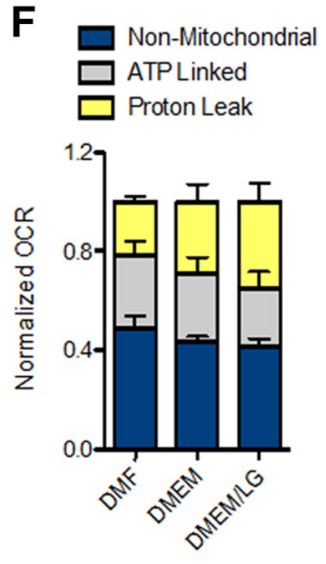

Figure 6. Human OLs decrease OXPHOS ATP production under metabolic stress conditions. $A, O C R$ in human OLs under the stated conditions demonstrates no significant changes in total OCR. B, ATP production was decreased by human OLs under low-glucose conditions. $\boldsymbol{C}$, Normalized mitochondrial oxygen consumption shows significant increases in proton leak with a significant decrease in ATP-linked OCR in low-glucose conditions compared with DFM medium. D, Human OPCs demonstrated no change in total $O C R$ under optimal and nutrient-deprived conditions. $\boldsymbol{E}$, No significant changes in ATP production were detected under the stated conditions. $\boldsymbol{F}$, No significant changes were detected in normalized OCR to nonmitochondrial, proton leak or ATP-linked oxygen consumption in optimal or stress conditions. ${ }^{*} p<0.05$.

higher glycolytic ATP production compared with OLs (Fig. 2D). The relative percentage of acidification for both nonglycolytic and glycolytic activities was similar between all cell types (Fig. 2E). This suggests that, in contrast to differences seen in normalized OCR for ATP synthesis, the amount of acidification due to glycolytic ATP production is similar across species.

\section{Total ATP production}

ATP is required for many cellular functions, including the DNA, protein, and lipid biosynthesis necessary for cell replication seen in OPCs and myelin biosynthesis seen in OLs. The rate of production and resulting consumption of ATP that we measured via the extracellular flux analyzer corresponds to the ATP required for these cellular processes. Calculation of total ATP production, the sum of OXPHOS and glycolysis, for the four cell types revealed lower rates of ATP production for human cells compared with rodent cells (Fig. 3A); this was expected because they had consistently lower metabolic rates. Both rodent and human OLs produced less ATP compared with OPCs, confirming that they require less energy for cellular maintenance (Fig. $3 A$ ). Calculation of the total percentage of ATP produced by OXPHOS or glycolysis showed that human OLs and OPCs predominantly use glycolytic pathways to produce ATP, in contrast to the rodent cells, which use OXPHOS (Fig. 3B). Furthermore, the relative percentage of the energetic sources used (OXPHOS vs glycolysis)

in the human and rodent cells appeared to be comparable during differentiation from OPCs to OLs (Fig. 3B).

\section{Sublethal injury studies}

The local environment of an MS lesion exposes OLs to hypoxia and diminished nutrients (Lassmann, 2003). Therefore, we investigated the metabolic changes that occur in adult human OLs and OPCs when challenged with conditions that mirror MS lesions. To accomplish this, cells were cultured under selected optimal or suboptimal conditions for up to $6 \mathrm{~d}$ (Fig. 4A). When left in the metabolic stress condition for $6 \mathrm{~d}$, both OLs and OPCs significantly increased their cell death rate, as seen by the reduced cell number (Fig. 4A), increased percentage of $\mathrm{PI}^{+}$staining (Fig. $4 B$, a marker for cell death), and percentage of TUNEL ${ }^{+}$staining (Fig. $4 C$, a marker for apoptosis), compared with cells maintained under optimal conditions. The extent of $\mathrm{PI}^{+}$cells was greater than TUNEL $^{+}$cells (Fig. 4B,C), suggesting that, for both OLs and OPCs, cell death may involve multiple mechanisms.

From these results, we identified that the $2 \mathrm{~d}$ treatment of the stated metabolic insults would provide a model of the initial sublethal conditions implicated in MS lesions. OLs cultured in DMEM/LG for $48 \mathrm{~h}$ showed no increase in the percentage of $\mathrm{PI}^{+}$cells and a marginal increase in $\mathrm{TUNEL}^{+}$cells compared with DFM or DMEM, with no change in total cell number (Fig. $4 D$ ). Cell process area per cell decreased significantly in DMEM/LG compared with DMEM (Fig. 4E,F). Time-lapse imaging studies (VivaView) showed process retraction of individual cells and cell aggregation under the DMEM/LG condition, in contrast to the cell process elongation under DFM control conditions (Fig. 5A,B). JC-1 staining showed that the mitochondria membrane potential was significantly depolarized under DMEM-LG conditions compared with DFM (Fig. 5C,D), indicating mitochondrial stress. The cells under DMEM/LG conditions had increased aggregation of LC3B, an indicator of autophagy activation (Fig. $5 E, F$ ), a cell-protective response initiated in response to nutrient deprivation. To continue mirroring the local environment of MS, we introduced hypoxia condition in our studies. Hypoxia alone did not induce measurable changes in the percentage of $\mathrm{TUNEL}^{+}$cells or cell numbers, but did result in reduced process outgrowth compared with cells in DMEM (Fig. 4E,F). Even the combination of DMEM/LG and hypoxia did not produce a significant effect on cell death compared with either insult alone, but did result in an increase in process retraction (Fig. $4 E$ ).

OPCs cultured for $48 \mathrm{~h}$ in DMEM/LG showed a slight increase in percentage of $\mathrm{PI}^{+}$and $\mathrm{TUNEL}^{+}$cells compared with DFM conditions, but no overall changes in total cell numbers. Under DFM conditions, OPCs had more robust process extension compared with OLs (Fig. 4E,F). Under DMEM/LG conditions, there was greater relative process retraction in OPCs (Fig. 4E, F). Hyp- 

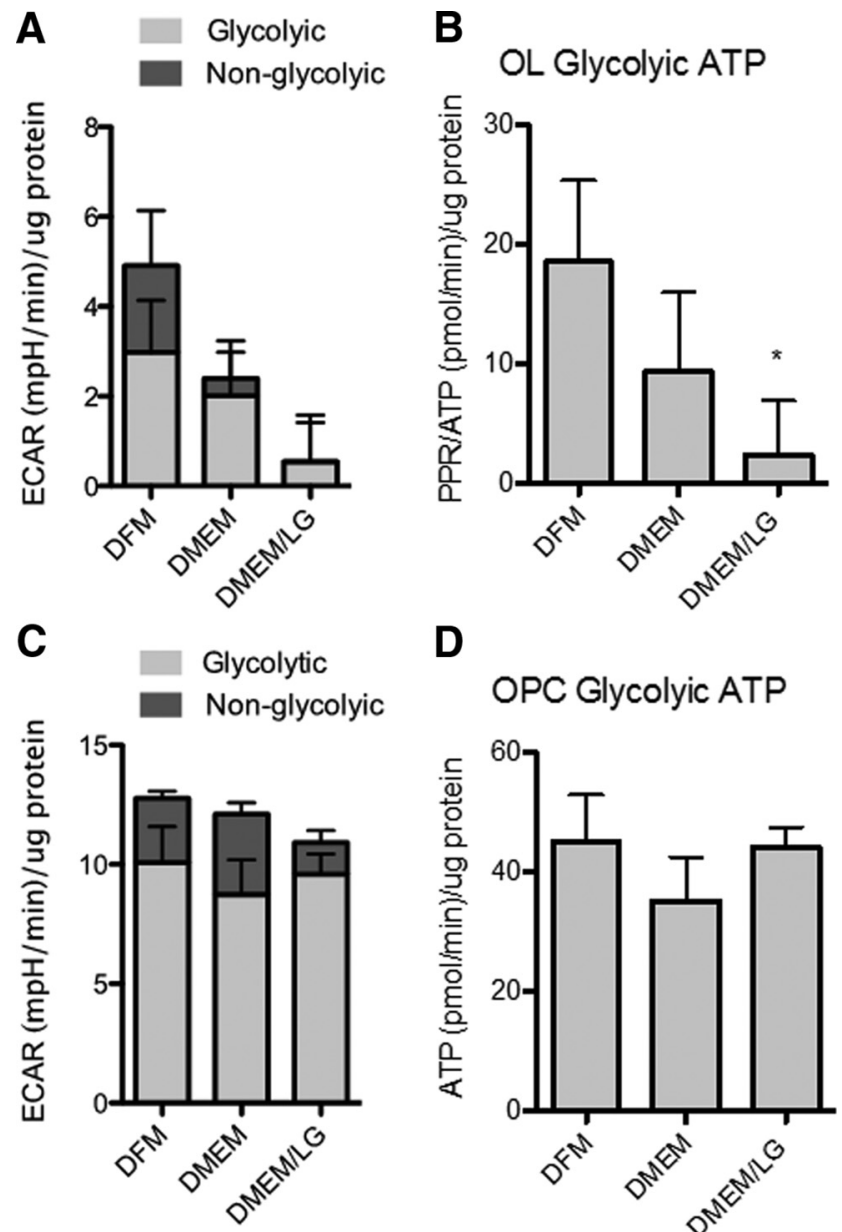

D
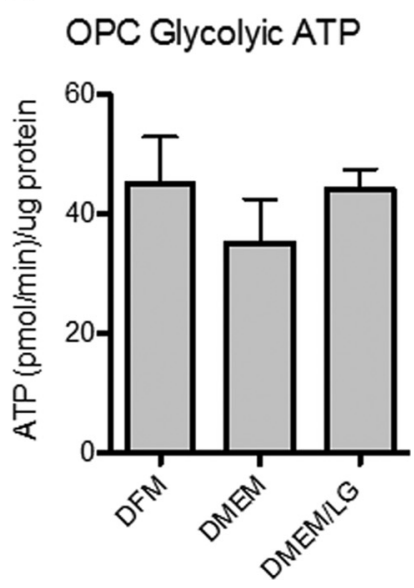

Figure 7. Human OLs significantly decrease glycolysis under metabolic stress conditions. $\boldsymbol{A}, \mathrm{ECAR}$ rates for human $\mathrm{OL}$ decrease under DMEM and low-glucose conditions compared with DFM. $\boldsymbol{B}$, This decrease resulted in a significant decrease in ATP production under low-glucose conditions. $\boldsymbol{C}$, Human OPCs demonstrated no significant decrease in ECAR under low-glucose conditions. D, There was no significant change in ATP production under low-glucose conditions compared with DFM.

oxia alone produced effects in OPCs similar to those seen in OLs, with no increase in cell death but an increase in process retraction (Fig. 4E). For OPCs, as for the OLs, there was evidence both of reduced mitochondrial membrane potential and increase autophagy (Fig. 5C-F).

\section{Bioenergetic properties in metabolic stress conditions}

Having identified appropriate conditions of sublethal stress, we then examined the human OLs and OPCs after $48 \mathrm{~h}$ in enriched media (DFM), DMEM, DMEM/LG, and also a $1 \% \mathrm{O}_{2}$ hypoxic condition. Although technical limitations prevent extracellular flux analysis under sustained hypoxic conditions, our analysis after $1 \%$ hypoxia culture showed enhanced total OCR compared with control conditions, consistent with cell survival and a metabolic rebound effect (data not shown).

\section{Altered OCR and ECAR under stress conditions}

For OLs, under the increasing stress condition of nutrient withdrawal, a minor decrease of OCR was detected relative to the already low rate of overall oxygen consumption (Fig. 6A). This decrease in OCR was primarily due to a reduction in mitochondrial ATP linked OCR, revealed by the corresponding decrease in ATP production (Fig. 6B). Normalization of OCR revealed that
OLs use less oxygen for ATP production and significantly more oxygen is consumed via proton leak when cultured with low glucose compared with DFM (Fig. 6C). Proton leak can be induced as a stress response to prevent the generation of free radicals (Brookes, 2005), confirming the stressed profile of the OLs.

In comparison, OPCs maintained higher basal OCRs compared with OLs, with no significant differences in OCR between conditions (Fig. 6D). Compared with OLs, OPCs did not alter oxygen utilization, with no changes in ATP linked OCR or ATP produced in conditions of increasing stress (Fig. 6E). Consistent with this, normalized data demonstrated no significant change in OCR utilization under any of the three conditions (Fig. 6F). These findings suggest that OPCs are unable to reduce their metabolic rate to promote cell survival under nutrient-deprived conditions.

Analysis of the glycolytic activity of human OLs showed a decrease in ECAR in DMEM/LG compared with DFM (Fig. 7A), with a significant decrease in ATP production (Fig. $7 B$ ). The OPCs once again showed no significant change in ECAR under low-glucose conditions (Fig. 7C) and no change in glycolytic ATP production (Fig. 7D). In low-glucose conditions, OLs were expected to decrease their glycolytic rate, resulting in less ATP production, but, as was observed for OPCs, the low level of glucose appeared to be sufficient to maintain glycolysis and not alter ATP production.

\section{Altered ATP production}

Human OLs decreased the level of ATP produced from OXPHOS and glycolysis under conditions of increased stress, whereas OPCs did not (Fig. $8 A, B$ ). The majority of the decreased OL ATP production was due to reduced glycolysis, with no change in either the rate of OXPHOS or glycolysis in OPCs. The results here confirm earlier observations that OLs are less metabolically active than OPCs and exhibit greater capacity to decrease ATP production rates when challenged by metabolic stress. In contrast, more metabolically active OPCs do not exhibit this capacity to alter their energetic profile, even after decreasing process extension, resulting in an increase in cell death rate compared with OLs.

\section{Discussion}

We used extracellular flux analysis to characterize the metabolic properties of human adult brain-derived OLs, comparing basal conditions with metabolic stresses that induce process retraction, modeling a recognized MS pathology. We demonstrated that human OLs are less metabolically active than OPCs derived from the same tissue and both are less active than OLs and OPCs derived from postnatal rats. The decrease in metabolic activity for the human cells in both OCR and ECAR results in significantly less ATP production per microgram of tissue for human cells compared with rodents. This could reflect species differences and/or the developmental state of the cells at the time of tissue collection. Although the overall metabolic rate of humans has been demonstrated to be lower than in rats (Speakman, 2005), we propose that the developmental state of the tissue plays a larger role in this difference, as was observed previously through in vivo work (Fünfschilling et al., 2012), but more experiments are necessary to confirm this observation.

Our analysis of the mechanisms of energy production indicates that human OLs and OPCs generate the majority of ATP from glycolysis, whereas the generation of ATP by rodent cells is predominantly dependent on OXPHOS. Aerobic glycolysis generates ATP with less efficiency than OXPHOS, but, importantly, 
A OL ATP Production

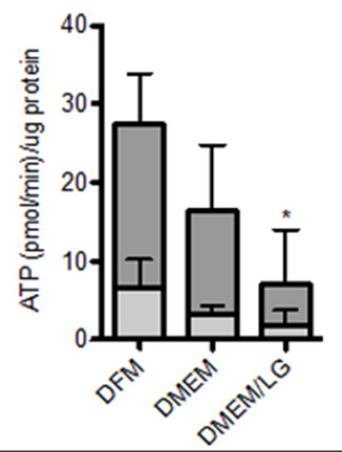

B OPC ATP Production

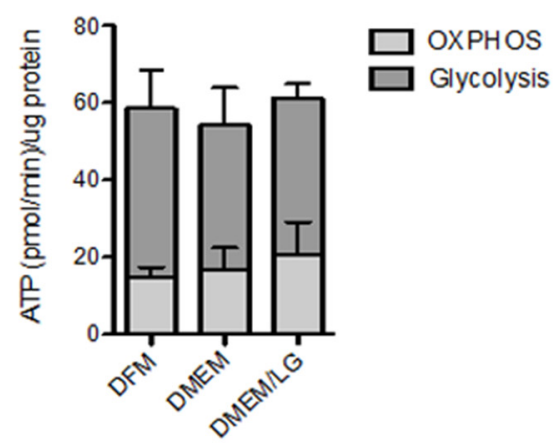

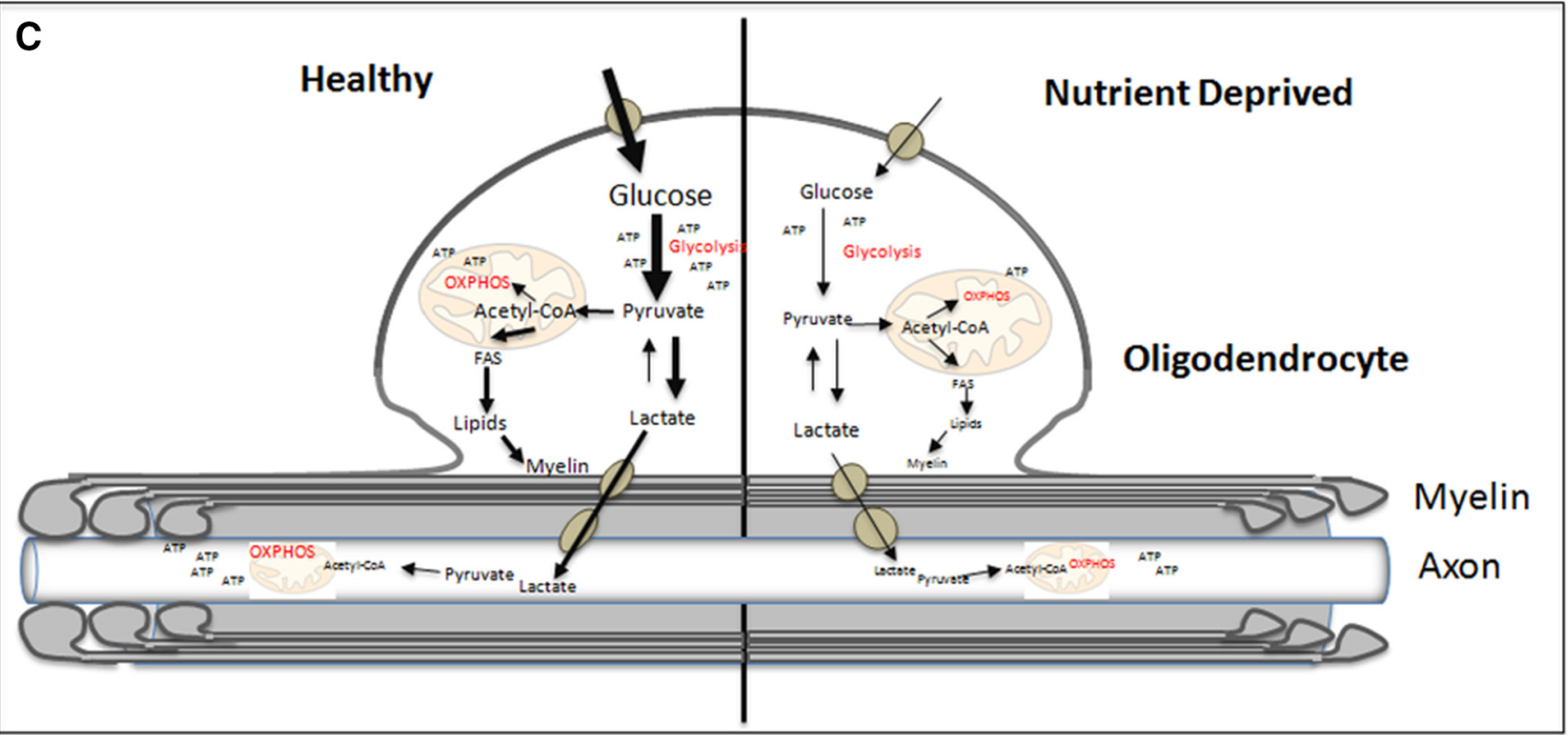

Figure 8. Human $0 \mathrm{Ls}$ decrease energetic requirements when stressed but OPCs do not adapt. $A$, Significant decrease in total ATP production seen with the decrease in nutrients with human OLs. $B$, No significant decrease in total ATP production was detected with nutrient reduction in human OPCS. C, Under normal conditions, human OLs undergo a low rate of OXPHOS ATP production with increased rates of glycolytic ATP production, a process that has demonstrated to be beneficial for myelin biosynthesis and also for lactic acid transfer to neurons. Under metabolic stress conditions, OLs decrease glycolytic and OXPHOS ATP production, resulting in process retraction and decreased lactic acid production. (Figure adapted from Fünfschilling et al., 2012.)

produces the carbon chain precursors necessary to support protein and lipid biosynthesis for myelin production (Bauernfeind et al., 2014). Notably, utilization of the glycolytic pathway would limit the production of ROS, reducing oxidative damage to the DNA (Brand and Hermfisse, 1997) and promoting the production of long-lived lipids required by OL myelin (Bongarzone et al., 1995). A high rate of glycolysis also promotes a high level of lactate production and release into the extracellular environment. Recent studies have documented the ability of OLs to transfer lactate to axons, where it is used as an energy source, metabolically supporting the axons (Lee et al., 2012). Therefore, the preference of aerobic glycolysis by long-lived adult human OLs would be beneficial under basal conditions to both the maintenance of OLs and the metabolic support of neurons. Because our current in vitro observations using isolated OLs and OPCs demonstrate the adaptability of glycolytic rates under stress conditions, in vivo rates are also likely not fixed because they would respond both to myelin and axonal metabolic demands. Carbon 14 dating demonstrates a high turnover rate of myelin with increased production in response to physiologic experience and these processes are dependent on the metabolic activity of existing OLs (Yeung et al., 2014).
We then focused our studies of the bioenergetic responses of OLs to metabolic stress on conditions that model the dying-back pathology seen in MS. This resulted in process retraction with marginal levels of cells death, whereas prolonged exposure promoted cell death that modeled the cell loss that occurs over time in MS. After $2 \mathrm{~d}$ of treatments, the OLs showed a significant decrease in the rate of glycolysis and process retraction (Figs. $8 B$, $4 C)$. We propose that this reflects an attempt by the cell to favor survival while reducing energetic demands for membrane production and maintenance (Fig. $8 C$ ). This sacrifice of the "luxury function" of myelin synthesis and maintenance by OLs would be extremely detrimental to neurons because the OLs have extensive processes with each cell in vivo, wrapping up to 50 axonal internodes. Furthermore, the reduced rates of glycolysis would decrease lactate transfer to axons, decreasing their ability to produce ATP (Fünfschilling et al., 2012). Because reduced transfer of lactate would theoretically occur before myelin retraction, this could provide one mechanism for axonal damage seen without the demyelination that occurs in MS (Metz et al., 2007; Fig. 8C). Multiple molecular pathways could be involved in linking the metabolic and phenotypic changes that we observed and several of these have been shown to be disrupted in MS, including glu- 
cose transporter, hexokinases, and members of the mitochondrial electron transport chain (Kalman et al., 2007; Kim et al., 2013). We demonstrate that our in vitro stress conditions are associated with reductions in mitochondrial membrane potential, an indicator of mitochondrial stress, and activation of autophagy. Directly translating our in vitro findings to the in vivo situation is complex because the levels of oxygen (21\%) and glucose (17 mM) used to culture our isolated populations of OLs under optimal culture conditions (DMF) differ significantly from conditions in the brain (i.e., oxygen $0.5 \%$ to $>7 \%$, Hutchinson et al., 2014; glucose $2-3 \mathrm{mmol} / \mathrm{L}$, Rostami and Bellander, 2011), where multiple other cell types and buffering systems are present.

Our results show that nutrient starvation conditions are more detrimental to the survival of the more metabolically active OPCs than for OLs. The human OPCs show increased TUNEL ${ }^{+}$cell death under basal conditions compared with the OLs, with further increased death when metabolically stressed (Fig. 4A-C). Although process extension was decreased by OPCs when stressed, similar to OLs, this was not sufficient to promote cell survival to the same extent as documented for OLs (Fig. 4A-C). We reported previously a relatively greater proportional loss of OPCs compared with OLs within active MS lesions, with increased OPCs in the surrounding area (Cui et al., 2013), consistent with the local environment of the lesion being detrimental to OPC survival. The altered metabolic profile seen in MS is predicted to contribute both to impaired myelin maintenance and the OPCs' capacity to replace the ensuing myelin loss.

\section{Conclusion}

The distinct energetic property of OLs under our model of metabolic stress conditions reflects the cells' attempt to favor survival while reducing demands for membrane production and maintenance. This contributes to the dying-back oligodendrogliopathy observed in active MS lesions and eventually the consequent OL loss that occurs over the prolonged disease course. The increased bioenergetic property of the OPCs contributes to their enhanced susceptibility to metabolic stress injury, as is postulated to occur in acute and chronic MS lesions, preventing remyelination. These distinct properties underlie the development of the dying-back oligodendrogliopathy that can be observed in active MS lesions and to the loss of OLs that continues throughout the subsequent progressive phase of the disease. Previous studies have shown that increasing glycolytic rates can increase myelin biosynthesis in OLs (Steiner et al., 2014). A wide array of compounds in clinical use are available for targeting the glycolytic pathway, raising the possibility that they could be applied to promote OL cell survival and to enhance myelin maintenance and repair in MS.

\section{References}

Aboul-Enein F, Rauschka H, Kornek B, Stadelmann C, Stefferl A, Brück W, Lucchinetti C, Schmidbauer M, Jellinger K, Lassmann H (2003) Preferential loss of myelin-associated glycoprotein reflects hypoxia-like white matter damage in stroke and inflammatory brain diseases. J Neuropathol Exp Neurol 62:25-33. CrossRef Medline

Almazan G, Afar DE, Bell JC (1993) Phosphorylation and disruption of intermediate filament proteins in oligodendrocyte precursor cultures treated with calyculin A. J Neurosci Res 36:163-172. CrossRef Medline

Amaral AI, Meisingset TW, Kotter MR, Sonnewald U (2013) Metabolic aspects of neuron-oligodendrocyte-astrocyte interactions. Front Endocrinol (Lausanne) 4:54. CrossRef Medline

Bauernfeind AL, Barks SK, Duka T, Grossman LI, Hof PR, Sherwood CC (2014) Aerobic glycolysis in the primate brain: reconsidering the implications for growth and maintenance. Brain Struct Funct 219:1149-1167. CrossRef Medline
Bongarzone ER, Pasquini JM, Soto EF (1995) Oxidative damage to proteins and lipids of CNS myelin produced by in vitro generated reactive oxygen species. J Neurosci Res 41:213-221. CrossRef Medline

Brand KA, Hermfisse U (1997) Aerobic glycolysis by proliferating cells: a protective strategy against reactive oxygen species. FASEB J 11:388-395. Medline

Brand MD (2005) The efficiency and plasticity of mitochondrial energy transduction. Biochem Soc Trans 33:897-904. Medline

Brookes PS (2005) Mitochondrial H(+) leak and ROS generation: an odd couple. Free Radic Biol Med 38:12-23. CrossRef Medline

Chang A, Nishiyama A, Peterson J, Prineas J, Trapp BD (2000) NG2positive oligodendrocyte progenitor cells in adult human brain and multiple sclerosis lesions. J Neurosci 20:6404-6412. Medline

Cui QL, Kuhlmann T, Miron VE, Leong SY, Fang J, Gris P, Kennedy TE, Almazan G, Antel J (2013) Oligodendrocyte progenitor cell susceptibility to injury in multiple sclerosis. Am J Pathol 183:516-525. CrossRef Medline

Divakaruni AS, Paradyse A, Ferrick DA, Murphy AN, Jastroch M (2014) Analysis and interpretation of microplate-based oxygen consumption and $\mathrm{pH}$ data. Methods Enzymol 547:309-354. CrossRef Medline

Eisenbarth GS, Walsh FS, Nirenberg M (1979) Monoclonal antibody to a plasma membrane antigen of neurons. Proc Natl Acad Sci U S A 76: 4913-4917. CrossRef Medline

Fünfschilling U, Supplie LM, Mahad D, Boretius S, Saab AS, Edgar J, Brinkmann BG, Kassmann CM, Tzvetanova ID, Möbius W, Diaz F, Meijer D, Suter U, Hamprecht B, Sereda MW, Moraes CT, Frahm J, Goebbels S, Nave KA (2012) Glycolytic oligodendrocytes maintain myelin and longterm axonal integrity. Nature 485:517-521. Medline

Harris JJ, Attwell D (2012) The energetics of CNS white matter. J Neurosci 32:356-371. CrossRef Medline

Herst PM, Berridge MV (2007) Cell surface oxygen consumption: a major contributor to cellular oxygen consumption in glycolytic cancer cell lines. Biochim Biophys Acta 1767:170-177. CrossRef Medline

Hutchinson P, O'Phelan K; Participants in the International Multidisciplinary Consensus Conference on Multimodality M (2014) International multidisciplinary consensus conference on multimodality monitoring: cerebral metabolism. Neurocrit Care 21:S148-S158. Medline

Kalman B, Laitinen K, Komoly S (2007) The involvement of mitochondria in the pathogenesis of multiple sclerosis. J Neuroimmunol 188:1-12. CrossRef Medline

Keuper M, Jastroch M, Yi CX, Fischer-Posovszky P, Wabitsch M, Tschöp MH, Hofmann SM (2014) Spare mitochondrial respiratory capacity permits human adipocytes to maintain ATP homeostasis under hypoglycemic conditions. FASEB J 28:761-770. CrossRef Medline

Kim SG, Buel GR, Blenis J (2013) Nutrient regulation of the mTOR complex 1 signaling pathway. Mol Cells 35:463-473. CrossRef Medline

Lassmann H (2003) Hypoxia-like tissue injury as a component of multiple sclerosis lesions. J Neurol Sci 206:187-191. CrossRef Medline

Lee Y, Morrison BM, Li Y, Lengacher S, Farah MH, Hoffman PN, Liu Y, Tsingalia A, Jin L, Zhang PW, Pellerin L, Magistretti PJ, Rothstein JD (2012) Oligodendroglia metabolically support axons and contribute to neurodegeneration. Nature 487:443-448. CrossRef Medline

Leong SY, Rao VT, Bin JM, Gris P, Sangaralingam M, Kennedy TE, Antel JP (2014) Heterogeneity of oligodendrocyte progenitor cells in adult human brain. Ann Clin Transl Neurol 1:272-283. CrossRef Medline

Lucchinetti C, Brück W, Parisi J, Scheithauer B, Rodriguez M, Lassmann H (2000) Heterogeneity of multiple sclerosis lesions: implications for the pathogenesis of demyelination. Ann Neurol 47:707-717. CrossRef Medline

Ludwin SK, Johnson ES (1981) Evidence for a "dying-back" gliopathy in demyelinating disease. Ann Neurol 9:301-305. CrossRef Medline

Lunt SY, Vander Heiden MG (2011) Aerobic glycolysis: meeting the metabolic requirements of cell proliferation. Annu Rev Cell Dev Biol 27: 441-464. CrossRef Medline

Mahad DH, Trapp BD, Lassmann H (2015) Pathological mechanisms in progressive multiple sclerosis. Lancet Neurol 14:183-193. CrossRef Medline

Metz I, Lucchinetti CF, Openshaw H, Garcia-Merino A, Lassmann H, Freedman MS, Atkins HL, Azzarelli B, Kolar OJ, Brück W (2007) Autologous haematopoietic stem cell transplantation fails to stop demyelination and neurodegeneration in multiple sclerosis. Brain 130:1254-1262. CrossRef Medline

Rodriguez M, Scheithauer BW, Forbes G, Kelly PJ (1993) Oligodendrocyte 
injury is an early event in lesions of multiple sclerosis. Mayo Clin Proc 68:627-636. CrossRef Medline

Rostami E, Bellander BM (2011) Monitoring of glucose in brain, adipose tissue, and peripheral blood in patients with traumatic brain injury: a microdialysis study. J Diabetes Sci Technol 5:596-604. CrossRef Medline

Ruffini F, Chojnacki A, Weiss S, Antel JP (2006) Immunobiology of oligodendrocytes in multiple sclerosis. Adv Neurol 98:47-63. Medline

Sánchez-Abarca LI, Tabernero A, Medina JM (2001) Oligodendrocytes use lactate as a source of energy and as a precursor of lipids. Glia 36:321-329. CrossRef Medline

Speakman JR (2005) Body size, energy metabolism and lifespan. J Exp Biol 208:1717-1730. CrossRef Medline

Stadelmann C, Ludwin S, Tabira T, Guseo A, Lucchinetti CF, Leel-Ossy L, Ordinario AT, Brück W, Lassmann H (2005) Tissue preconditioning may explain concentric lesions in Balo's type of multiple sclerosis. Brain 128:979-987. CrossRef Medline

Steiner J, Martins-de-Souza D, Schiltz K, Sarnyai Z, Westphal S, Isermann B, Dobrowolny H, Turck CW, Bogerts B, Bernstein HG, Horvath TL, Schild L, Keilhoff G (2014) Clozapine promotes glycolysis and myelin lipid synthesis in cultured oligodendrocytes. Front Cell Neurosci 8:384. Medline

Trapp BD, Nave KA (2008) Multiple sclerosis: an immune or neurodegenerative disorder? Annu Rev Neurosci 31:247-269. CrossRef Medline

Trapp BD, Stys PK (2009) Virtual hypoxia and chronic necrosis of demyelinated axons in multiple sclerosis. Lancet Neurol 8:280-291. CrossRef Medline

Witte ME, Mahad DJ, Lassmann H, van Horssen J (2014) Mitochondrial dysfunction contributes to neurodegeneration in multiple sclerosis. Trends Mol Med 20:179-187. CrossRef Medline

Yeung MS, Zdunek S, Bergmann O, Bernard S, Salehpour M, Alkass K, Perl S, Tisdale J, Possnert G, Brundin L, Druid H, Frisén J (2014) Dynamics of oligodendrocyte generation and myelination in the human brain. Cell 159:766-774. CrossRef Medline

Ziabreva I, Campbell G, Rist J, Zambonin J, Rorbach J, Wydro MM, Lassmann H, Franklin RJ, Mahad D (2010) Injury and differentiation following inhibition of mitochondrial respiratory chain complex IV in rat oligodendrocytes. Glia 58:1827-1837. CrossRef Medline 